\title{
Pre-main sequence variables in young cluster Stock 18
}

\author{
Tirthendu Sinha*, Saurabh Sharma, Rakesh Pandey, Anil Kumar Pandey
}

Aryabhatta Research Institute of Observational Sciences (ARIES), Nainital, 263 002, India

\begin{abstract}
We have carried out multi-epoch deep $I$ band photometry of the open cluster Stock 18 to search for variable stars in star forming regions. In the present study, we identified 65 periodic and 217 non-periodic variable stars. The periods of most of the periodic variables are between 2 hours to 15 days and their magnitude varies between 0.05 to $0.6 \mathrm{mag}$. We have derived spectral energy distributions for 48 probable pre-main sequence variables. Their average age and mass are $2.7 \pm 0.3$ Myrs and $2.7 \pm 0.2 \mathrm{M}_{\odot}$, respectively.
\end{abstract}

\section{Introduction}

The evolution of pre-main sequence (PMS) stars involves a set of complex physical process such as evolution of circumstellar disks, accretion processes, bipolar jets, angular momentum etc. Young star clusters are ideal sites for studying these characteristics as plenty of them are found in these regions with a broad mass range and a spread in their ages (few Myrs). PMS objects are generally classified as T Tauri stars (TTSs) and Herbig Ae/Be stars. The TTSs have masses $\leq 3 \mathrm{M}_{\odot}$, whereas Herbig $\mathrm{Ae} / \mathrm{Be}$ stars have higher masses in the range 3-10 $\mathrm{M}_{\odot}$. Depending on the strength of $\mathrm{H} \alpha$ emissions, TTSs can be further classified as classical TTSs (equivalent width EW $>10 \AA$ ) and weak line TTSs (EW $\leq 10 \AA$ ). The PMS stars usually show a wide range of variability in their luminosity in almost all wavelengths from X-ray to infrared and their variability time scales range from a few minutes to years (Appenzeller \& Mundt 1989). Several mechanisms are known to induce the photometric variability of PMS stars: irregular distribution of cool spots on stellar photosphere, variable hot spots, obscuration from dust (Herbst et al. 1994 and reference therein), instability in disk, change in accretion rate etc. The evolution of disks and the accretion rates may play a prominent role in the non-periodic variability (Hillenbrand 2002; Percy et. al. 2006, 2010), whereas due to the presence of cool and hot spots on the photosphere, rotation of the stars may produce periodic/quasi-periodic changes in their light curve (LC). These periods are a direct indicator of the rotational speed and hence they are related to the angular momentum. As the PMS stars contract towards the main sequence (MS), they should increase their rotation speed up to the break-up velocity due to conservation of angular momemtum. But numerous studies on PMS stars have revealed that these stars typically rotate at much smaller fraction of the break-up velocity in spite of their significant contraction. Several mechanisms to effectively remove angular momentum from PMS stars during the first $~ 10$ Myrs of their evolution have been proposed including magnetic star-disk interaction (disk-locking; Koenigl 1991; Shu et al. 1994; Najita 1995; Ostriker \& Shu 1995), scaled-up solar-type magnetized winds perhaps driven by accretion (e.g., Matt \& Pudritz 2004, 2005a, 2005b, 2008a, 2008b), and scaled-up solar-type coronal

*tirthendu@aries.res.in 
Table 1: Log of the optical observations for Stock 18. For each night, the telescope used for the observations (Telescope) and for each photometric passband (Filter $I$ and Filter $V$ ) the number of observervations (\#) together with their exposure times in seconds (Texp) are given.

\begin{tabular}{|c|c|ccc|ccc|}
\hline Date & Telescope & \multicolumn{3}{|c|}{ Filter $I$} & \multicolumn{3}{|c|}{ Filter $V$} \\
& & $\#$ & & Texp & $\#$ & & Texp \\
\hline $30 / 09 / 2016$ & DFOT & 85 & $\times$ & $180 \mathrm{~s}$ & 3 & $\times$ & $180 \mathrm{~s}$ \\
\hline $21 / 10 / 2016$ & DFOT & 135 & $\times$ & $180 \mathrm{~s}$ & 3 & $\times$ & $180 \mathrm{~s}$ \\
\hline $11 / 11 / 2016$ & DFOT & 85 & $\times$ & $180 \mathrm{~s}$ & 3 & $\times$ & $180 \mathrm{~s}$ \\
\hline $26 / 11 / 2016$ & DFOT & 20 & $\times$ & $180 \mathrm{~s}$ & 3 & $\times$ & $180 \mathrm{~s}$ \\
\hline $17 / 12 / 2016$ & DFOT & 1 & $\times$ & $180 \mathrm{~s}$ & 1 & $\times$ & $180 \mathrm{~s}$ \\
\hline $23 / 12 / 2016$ & TRT-GAO & 16 & $\times$ & $240 \mathrm{~s}$ & & & \\
\hline $24 / 12 / 2016$ & TRT-GAO & 26 & $\times$ & $240 \mathrm{~s}$ & & & \\
\hline $26 / 12 / 2016$ & DFOT & 1 & $\times$ & $180 \mathrm{~s}$ & & & \\
\hline
\end{tabular}

mass ejections (e.g., Aarnio et al. 2009, 2010, 2011). These mechanisms are still under debate and it is not clear yet to what extent each individual component contributes. The study of correlation between the rotation period and different stellar properties (age, mass, accretion rate etc.) is important to understand the angular momentum as well as the overall evolution of the PMS stars. The young open cluster Stock 18 with a cluster age of $6 \mathrm{Myr}$ (Bhatt et al. 2012) harbors a large number of PMS stars with a span in their age and mass. Therefore, it is an ideal site to look for PMS stars and study their properties. Here, we are presenting our preliminary analyses of the time series photometry of stellar sources in this region.

\section{Observation and data analysis}

Optical photometric observations of Stock 18 were taken with the 1.3-m Devasthal Fast Optical Telescope (DFOT; Devasthal, India) and the 0.7-m TRT-GAO Telescope (TRT-GAIO; Gao mei Gu Observatory, China). Both the telescopes have a $2048 \times 2048$ pixel square CCD camera but with different plate scales. The 1.3-m Devasthal telescope covers $\sim 18.4 \times 18.4 \mathrm{arcmin}^{2}$ field in the sky whereas the 0.7 -m telescope has a $\sim 20.9 \times 20.9 \mathrm{arcmin}^{2}$ field of view. In total, 369 and 13 frames were taken in $I$ and $V$ filters, respectively. Details of the observations are given in Table 1.

The basic image processing such as bias subtraction and flat fielding were done by using tasks available within IRAF ${ }^{1}$. The instrumental magnitude was obtained by using the DAOPHOT (Stetson 1987) package. As the cluster regions are very crowded, we carried out PSF photometry to get the magnitude of the stars. We have used the DAOMATCH and DAOMASTER routine of DAOPHOT-II (Stetson 1992) to estimate the shifts in the individual frames with respect to a reference frame and to get magnitudes of stars detected in different frames. The first frame of 21 October 2016 has been taken as reference. The observations obtained with the $1.3-\mathrm{m}$ and $0.7-\mathrm{m}$ telescope are cross matched by using their astrometry. Here we would like to point out that fainter stars detected with the 1.3-m telescope are not necessarily detected in the images taken with the 0.7-m telescope. We calibrated the instrumental magnitude to the standard system using the photometric data of Bhatt et. al. (2012).

\footnotetext{
${ }^{1}$ IRAF is distributed by the National Optical Astronomy Observatory, which is operated by the Association of Universities for Research in Astronomy (AURA) under cooperative agreement with the National Science Foundation.
} 

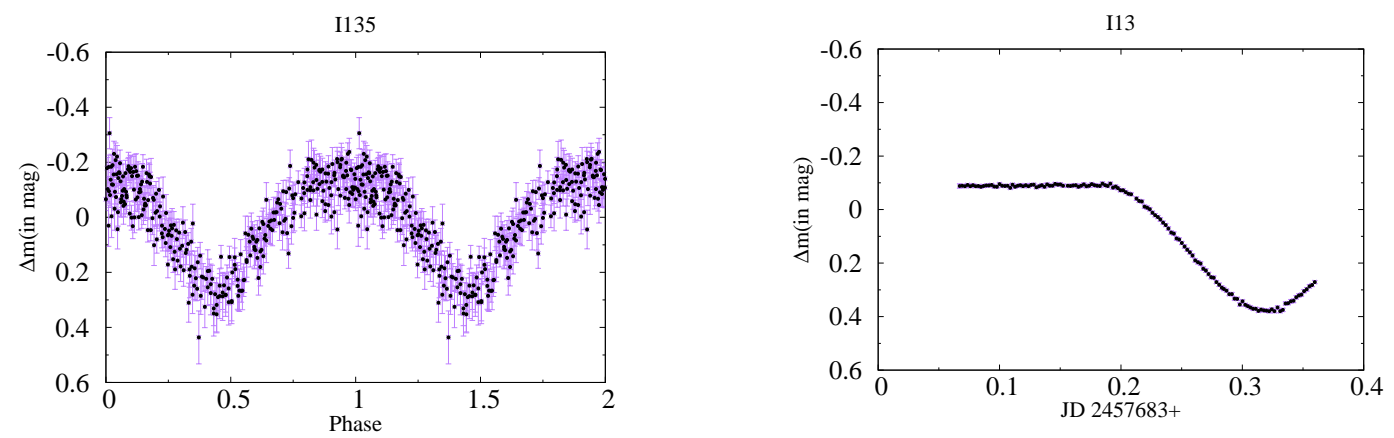

Figure 1: (left) Phase diagram for the periodic variable I135 whose LC was folded with a period of 4.5 hours. (right) LC for the non-periodic variable I13.

\section{Generation of light curve}

To generate the LCs of the sources, we have used different comparison and check stars in different magnitude bins (e.g., 10-11, 11-12, 12-13 mag and so on). To do so, we have taken all possible combinations of stars as potential comparison and check stars in a magnitude bin. Then we have calculated the standard deviation (SD) of the difference of magnitudes for all the combinations and the combination having the smallest SD is taken as comparison and check stars for that magnitude bin. The differential magnitude $(\Delta \mathrm{m})$ in the sense star-comparison were then plotted as a function of Julian date to generate the LC of a star. The probable variables were identified by visual inspection of these LCs. To determine the period of the probable variable stars, we used the Period ${ }^{2}$ software. The periodic variables are folded according to their period and plotted against their phase.

We have identified 65 periodic and 217 non-periodic variables in the region up to 20 mag in the $V$-band. Light curves of a few variables are shown in Fig.1. Most of the periodic variables have periods ranging from 2 hours to 15 days and their magnitude varies between 0.05 and 0.6 mag.

\section{Result and Conclusion}

The evolutionary status of these variables was explored through their position in the Hertzsprung Russell (HR) diagram (for details, see Sharma et al. 2017). Age and mass isochrones (Marigo et al. 2008 \& Siess et al. 2000) are used to determine the age and mass of a star. Out of the detected variables, 247 have good photometric data (error $<0.1 \mathrm{mag}$ ) and 130 of them have ages $>10 \mathrm{Myr}$. The later ones are most probably MS or field stars. The average age of the remaining variables are found to be $4.2 \pm 0.4$ Myrs (117 stars). Optical HR diagrams usually show a large spread at the fainter end and it is very difficult to differentiate between a MS star and a PMS star of higher age. Therefore, we have tagged a variable as probable PMS star only if its age is less than the average age of the selected sample and a more precise age has been derived by using the Spectral Energy Distribution (SED) fitting tool of Robitaille et al. (2007). The SED for 48 probable PMS-variables (5 of them are also showing an infrared excess) were generated by using the available optical, near-infrared (from 2MASS data archive ${ }^{3}$ ) and mid-infrared (from WISE data archive ${ }^{4}$ ) data, with a condition that they should have photometric data in at least 5 broad band filters. From the SED fitting analyses (for

\footnotetext{
${ }^{2}$ http://www.starlink.rl.ac.uk/docs/sun167.htx/sun167.html

${ }^{3}$ http://irsa.ipac.caltech.edu/Missions/2mass.html

${ }^{4} \mathrm{http}: / /$ irsa.ipac.caltech.edu/Missions/wise.html
} 

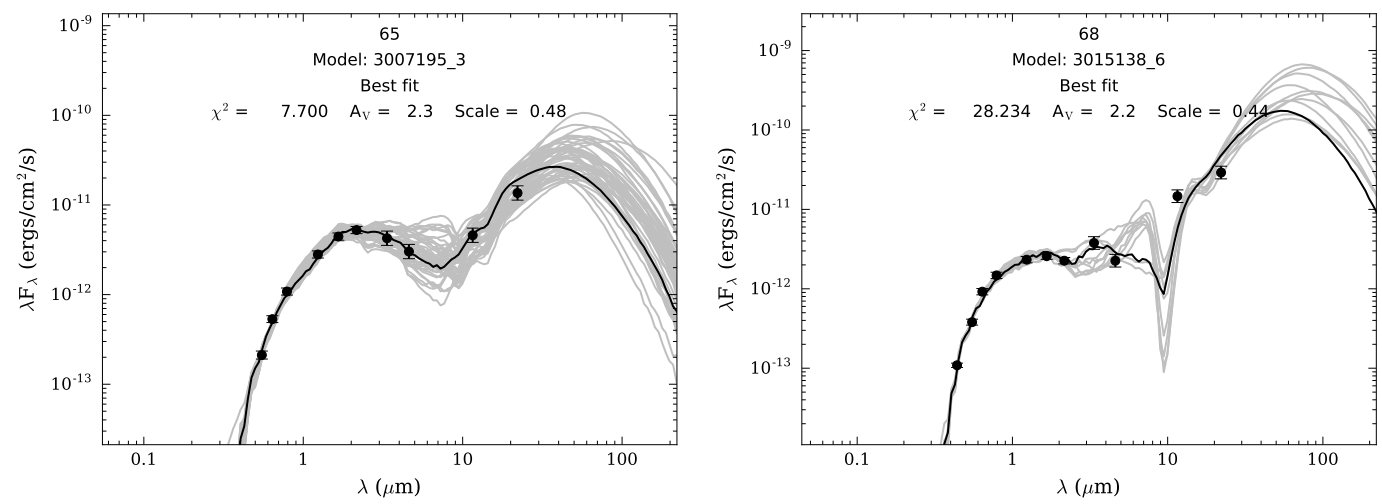

Figure 2: Sample SEDs for the PMS variable stars I65 (left) and I68 (right) created by the SED fitting tools of Robitaille et al. (2007) as an example to illustrate the quality of the results. The black curve shows the best fit and the grey curves show the subsequent good fits. The filled circles with error bars denote the input flux values.

details, cf. Sharma et al. 2017; see Fig.2 for some examples), the average age and mass of the probable PMS-variables is found to be $2.7 \pm 0.3$ Myrs and $2.7 \pm 0.2 \mathrm{M}_{\odot}$, respectively. This suggests that these stars should be T-Tauri stars. Disk/envelop accretion rate and disk mass of these PMSvariable stars are also being determined from the SED fitting. A detailed analysis is underway.

In this study, we have detected variable stars up to a mean mass of $2.7 \mathrm{M}_{\odot}$. We therefore can expect a large number of lower mass variables to be undetected in our survey. Detecting them will certainly enrich the statistics and help to understand disk/envelop evolution processes in the lower mass PMS regime. In the future, we will be using the recently installed 3.6-m Devasthal Optical Telescope (DOT) facility. With the optical imager we should be able to identify low mass variables and the TIFR+ARIES near-infrared spectrograph can be used for the follow-up of interesting variables.

\section{References}

Aarnio A. N., Stassun K. G., Hughes W. J. et. al. 2011, SoPh, 268, 195

Aarnio A. N., Stassun K. G., Matt S. P. 2010, ApJ, 717, 93

Appenzeller I., Mundt R. 1989, A\&AR, 1, 291

Bhatt H., Sagar R., Pandey J. C. 2012, NewA, 17,160

Herbst W., Herbst D. K., Grossman E. J. et. al. 1994, AJ, 108, 1906

Hillenbrand L. A. 2002, astro-ph/0210520

Koenigl A. 1991, ApJ, 370, L39

Marigo P., Girardi L., Bressan A. et. al. 2008, A\&A, 482,883

Matt S., Pudritz R. E. 2004, ApJ, 607, L43

Matt S., Pudritz R. E. 2005a, ApJ, 632, L135

Matt S., Pudritz R. E. 2005b, MNRAS, 356, 167

Matt S., Pudritz R. E. 2008a, ApJ, 678, 1109

Matt S., Pudritz R. E. 2008b, ApJ, 681, 391

Najita J. 1995, RMxAC, 1, 293

Ostriker E. C., Shu F. H. 1995, ApJ, 447, 813

Percy J. R., Gryc W. K., Wong J., Herbst W. 2006, PASP, 118, 1390

Percy J. R., Grynko S., Seneviratne R., Herbst W. 2010, PASP, 122, 753

Robitaille T. P., Whitney B. A., Indebetouw R., Wood K. 2007, ApJS, 169, 328

Sharma S., Pandey A. K., Ojha D. K. et al. 2017, MNRAS, 467, 2943

Shu F., Najita J., Ostriker E. et al. 1994, ApJ, 429, 781

Siess L., Dufour E., Forestini M. 2000, A\&A, 358, 593 